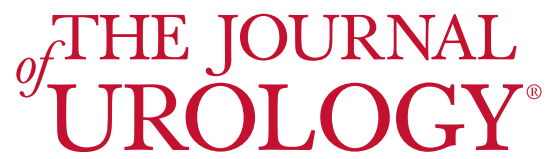

www.auajournals.org/journal/juro

\title{
Is the Toxicity of Salvage Prostatectomy Related to the Primary Prostate Cancer Therapy Received?
}

\author{
Luis Ribeiro, Thomas Stonier, Luke Stroman, Rafael Tourinho-Barbosa, Omar Alghazo, \\ Mathias Winkler, Prokar Dasgupta, Rick Popert, Xavier Cathelineau, Rafael Sanchez-Salas, \\ Declan G. Murphy, Mark Emberton* and Paul Cathcart†
}

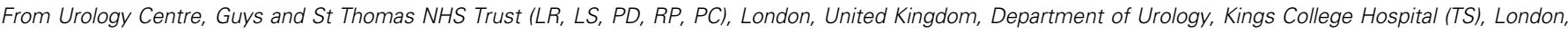
United Kingdom, Deptartment of Urology, Institut Mutualiste Montsouris, Université Paris-Descartes (RT-B, XC, RS-S), Paris, France, Division of Cancer Surgery, Sir Peter MacCallum Cancer Centre (OA, DGM), Melbourne, Australia, Imperial College NHS Foundation Trust (MW), London, United Kingdom, University College London Hospitals NHS Trust (ME), London, United Kingdom
\end{abstract}

Purpose: To compare the toxicity profile and oncological outcome of salvage radical prostatectomy following focal therapy versus salvage radical prostatectomy after radiation therapies (external beam radiation therapy or brachytherapy).

Materials and Methods: Data concerning all men undergoing salvage radical prostatectomy for recurrent prostate cancer after either focal therapy, external beam radiation therapy or brachytherapy were retrospectively collected from 4 high volume surgical centers. The primary outcome measure of the study was toxicity of salvage radical prostatectomy characterized by any 30-day postoperative Clavien-Dindo complication rate, 12 -month continence rate and 12 -month potency rate. The secondary outcome was oncological outcome after salvage radical prostatectomy including positive margin rate and 12-month biochemical recurrence rate. Biochemical recurrence was estimated using Kaplan-Meier methods and significant differences were calculated using a log rank test. Median followup was 29.5 months.

Results: Between April 2007 and September 2018, 185 patients underwent salvage radical prostatectomy of whom 95 had salvage radical prostatectomy after focal therapy and 90 had salvage radical prostatectomy after radiation therapy (external beam radiation therapy or brachytherapy). Salvage radical prostatectomy after radiation therapy was associated with a significantly higher 30 -day Clavien-Dindo I-IV complication rate ( $34 \%$ vs $5 \%, \mathrm{p}<0.001)$. At 12 months following surgery, patients undergoing salvage radical prostatectomy after focal therapy had significantly better continence (83\% pad-free vs $49 \%$ ) while potency outcomes were similar (14\% vs $11 \%$ ). Men undergoing salvage radical prostatectomy after radiation therapy had a significantly higher stage and grade of disease together with a higher positive surgical margin rate ( $37 \%$ vs $13 \%, \mathrm{p}=0.001$ ). The 3 -year biochemical recurrence after focal therapy was $35 \%$ compared to $32 \%$ after radiation therapy $(p=0.76)$. In multivariable analysis, men undergoing salvage radical prostatectomy after focal therapy experienced a higher risk of biochemical recurrence (HR 0.36, 95\% CI 0.16-0.82, $\mathrm{p}=0.02$ ).

Conclusions: This multicenter study demonstrates the toxicity of salvage radical prostatectomy in terms of perioperative complications and long-term urinary continence recovery is dependent on initial primary prostate cancer therapy received with men undergoing salvage radical prostatectomy after

\section{Abbreviations and Acronyms \\ $\mathrm{BCR}=$ biochemical recurrence \\ $\mathrm{BT}=$ brachytherapy \\ EBRT $=$ external beam \\ radiotherapy \\ $\mathrm{FT}=$ focal therapy \\ HIFU = high intensity focused ultrasound \\ ISUP $=$ International Society of Urological Pathology \\ PSA $=$ prostate specific antigen \\ $\mathrm{RT}=$ radiation therapy \\ $\mathrm{SRP}=$ salvage radical \\ prostatectomy}

Accepted for publication August 26, 2020

* Financial and/or other relationship with Sonacare, Sophiris Biocorp, Steba Biotech, Trod Medical and Immodulon.

† Correspondence: Guys and St Thomas NHS Foundation Trust, Urology Centre, London, London EC1M 6BO, United Kingdom (email: paul. cathcart@gstt.nhs.uk).
https://doi.org/10.1097/JU.0000000000001382 Vol. 205, 791-799, March 2021 Printed in U.S.A. 
focal therapy experiencing lower postoperative complication rates and better urinary continence outcomes.

Key Words: prostatic neoplasms; neoplasm recurrence, local; prostatectomy; outcome assessment, health care; quality of health care

NonSURGICAL treatments for prostate cancer include traditional radiation approaches such as external beam radiation therapy and brachytherapy, and new minimally invasive therapies including high intensity focused ultrasound, cryotherapy and electroporation. These newer focal therapies target only the location of the tumor within the prostate as opposed to irradiating the entire prostate. ${ }^{1}$ The proportion of men experiencing recurrent disease after EBRT or BT ranges from $10 \%$ to $50 \%$ at 10 years depending on disease stratification prior to radiation therapy. ${ }^{2}$ Recurrence after FT is difficult to quantify as it is newer, but reported rates range from $10 \%$ to $40 \%$ with up to $25 \%$ of men undergoing repeat FT or some form of salvage therapy. ${ }^{1,3,4}$

Salvage radical prostatectomy is a potential treatment option for carefully selected men experiencing local recurrence following RT or FT. ${ }^{2,5}$ Historically, most men experiencing disease recurrence after radiation therapy have only received palliative hormone therapy. The CaPSURE longitudinal observational study suggested less than $1 \%$ of men eligible for SRP after radiation treatment underwent SRP. ${ }^{6}$ The high reported toxicity of SRP together with the technical difficulties after radiation is a potential reason why so few undergo the procedure. ${ }^{7}$

Data concerning SRP after FT are more limited than SRP after radiation as FT is a newer treatment approach. SRP after RT for men experiencing disease recurrence has been associated with high toxicity. ${ }^{8,9}$ As FT is designed to cause less tissue damage at the time of therapy, the toxicity of SRP after FT could be hypothesized to be significantly less than after RT.

The aim of the current study was to compare the toxicity profile and oncological outcome of SRP following FT versus SRP after EBRT or BT.

\section{METHODS}

\section{Study Population}

We performed a multicenter retrospective cohort study of consecutive patients undergoing SRP for recurrent prostate disease between 2007 and 2019 across 4 high volume surgical centers: Guy's Hospital (London, UK), Institut Mutualiste Montsouris (Paris, France), Imperial College Healthcare Trust (London, UK) and The Peter MacCallum Cancer Centre (Melbourne, Australia) (supplementary table 1, https://www.jurology.com). The study had local Institutional Review Board approval at Guy's Hospital (IRB No. IORG0002231) and Institut Mutualiste Montsouris (IRB
No. IORG0010120). Data were collected as part of the British Association of Urological Surgeons and Royal Australasian College of Surgeons mandatory audit.

Group 1 included patients with recurrent prostate cancer after RT, either receiving primary EBRT or primary BT. Most patients had received their therapy at an external center and then referred to the surgical center for salvage therapy.

Group 2 consisted of patients undergoing SRP for recurrent prostate cancer after undergoing primary FT (defined as ablation of the index or dominant prostate cancer lesion). Patients underwent a combination of hemigland, pure focal or subtotal gland ablation (dogleg ablation) co-registered with preoperative magnetic resonance imaging findings and pre-therapy prostate mapping biopsy. Patients had a combination of in-field and out-of-field recurrent disease. The focal treatment centers have performed in excess of 2,000 treatments and have published their outcomes. ${ }^{10}$ Post-focal biopsy was selectively performed based on PSA dynamics and post-focal magnetic resonance imaging. Group 2 has previously been reported in a brief correspondence article ${ }^{11}$ albeit the data presented here have longer followup.

All patients were required to have a recent biopsy of the prostate after their initial primary treatment confirming recurrent prostate cancer prior to undergoing SRP. Metastatic disease was excluded in all patients prior to SRP using pelvic magnetic resonance imaging, bone scans, and/ or positron emission tomography/computed tomography. Patients were followed up at 8 weeks post-surgery and every 3 months thereafter. Details on our SRP surgical technique are documented in the supplementary Appendix (https://www.jurology.com).

\section{Study Outcomes}

The primary outcome of the study was the toxicity of SRP. We report both postoperative complications within the first 30 days of surgery according to the Clavien-Dindo grading system and functional outcome of SRP captured by a strict definition of urinary continence (patient reported use of no pads at 12 months after surgery) and a strict definition of potency (patient reported ability to perform spontaneous penetrative intercourse with or without the use of phosphodiesterase type- 5 inhibitor 12 months after surgery.

The secondary outcome of the study was the reporting of oncological outcome after SRP utilizing both pathological prostatectomy margin status (positive/negative) and biochemical recurrence at 3 years after surgery. RR was defined as a serum PSA $>0.2 \mathrm{ng} / \mathrm{ml}$.

\section{Statistical Analysis}

Statistical analysis was performed using SPSS ${ }^{\circledR}$ v25.0 (IBM SPSS Statistics for Windows, 2017, IBM 
Corporation, Armonk, New York). BCR-free survival was estimated using the Kaplan-Meier method. A Cox regression multivariate model was constructed to determine the impact of risk factors for BCR after salvage surgery and adjust for inherent baseline differences. Sensitivity analyses were also performed to compare patients with earlier salvage surgery (SRP within 5 years of primary therapy) and more contemporary cohorts (SRP performed after 2015).

\section{RESULTS}

Between April 2007 and November 2018, 90 men underwent SRP after RT, and between January 2010 and January 2019, 95 underwent SRP after FT. Patient characteristics are presented in table 1.

Primary treatment modalities are presented in table 2. Radiation doses were available for $45 \%$ of the patients. Median dose for EBRT was 64 Gy (IQR 55-70) and all

Table 1. Summary and comparison of oncological factors in focal therapy and radiotherapy salvage prostatectomies

\begin{tabular}{|c|c|c|c|}
\hline & SRP after RT/BT & SRP after FT & $\mathrm{p}$ Value \\
\hline No. pts & 90 & 95 & \\
\hline Median age at surgery (IOR) & $(62-70)$ & $(60-69)$ & 0.15 \\
\hline No. surgical technique (\%): & & & $<0.001$ \\
\hline Laparoscopic & (9) & (0) & \\
\hline Open & (9) & (0) & \\
\hline$\%$ Yr of procedure: & & & 0.002 \\
\hline 2009 & 3 & 0 & \\
\hline 2010 & 2 & 2 & \\
\hline 2011 & 2 & 1 & \\
\hline 2012 & 14 & 2 & \\
\hline 2013 & 8 & 1 & \\
\hline 2014 & 1 & 7 & \\
\hline 2015 & 8 & 15 & \\
\hline Mean pre-SRP PSA (range) & $7.0(0.06-34.0)$ & $7.1(0.3-24.0)$ & 0.89 \\
\hline$\%$ Pre-primary treatment ISUP group: & & & $<0.001$ \\
\hline 1 & 38 & 37 & \\
\hline 2 & 27 & 50 & \\
\hline 3 & 23 & 13 & \\
\hline 4 & 1 & 0 & \\
\hline 5 & 11 & 0 & \\
\hline ISUP group on pre-SRP biopsy: & & & $<0.001$ \\
\hline 1 & 3 & 11 & \\
\hline 2 & 30 & 52 & \\
\hline 3 & 23 & 30 & \\
\hline 4 & 16 & 3 & \\
\hline 5 & 18 & 3 & \\
\hline T3b & 8 & 2 & \\
\hline$\%$ Pre-SRP T stage: & & & $<0.001$ \\
\hline T1C & 4 & 22 & \\
\hline $\mathrm{T} 2$ & 60 & 64 & \\
\hline ТЗа & 16 & 4 & \\
\hline $\mathrm{T} 3 \mathrm{~b}$ & 16 & 10 & \\
\hline \% Pathological T stage: & & & 0.09 \\
\hline T2 & 36 & 46 & \\
\hline ТЗа & 27 & 31 & \\
\hline $\mathrm{T} 3 \mathrm{~b}$ & 38 & 23 & \\
\hline \% Pathological N stage: & & & $<0.001$ \\
\hline $\mathrm{pNx}$ & 59 & 74 & \\
\hline pNO & 31 & 12 & \\
\hline pN1 & 10 & 1 & \\
\hline
\end{tabular}


Table 2. Breakdown of primary therapy by type of energy used in focal therapy and radiotherapy salvage prostatectomies

\begin{tabular}{lr}
\hline Primary therapy & No. Pts $(\%)$ \\
\hline Focal Therapy: & $95(51)$ \\
HIFU & $65(68)$ \\
Cryotherapy & $17(18)$ \\
Electroporation (NanoKnife) & $8(8)$ \\
Vascular photodynamic therapy & $3(3)$ \\
Topsalysin PRX302 & $2(2)$ \\
Radiotherapy: & $90(49)$ \\
EBRT & $56(62)$ \\
High dose brachytherapy & $4(4)$ \\
Low dose brachytherapy & $27(30)$ \\
Cyberknife & $1(1)$ \\
EBRT + brachytherapy & $2(2)$ \\
\hline
\end{tabular}

patients undergoing BT received 145 Gy. Concomitant hormone therapy was given to all patients undergoing EBRT while no patient undergoing BT received concomitant hormone therapy. Median time from primary therapy to SRP following FT or RT was 3 years (IQR 2-5) and 7 years (IQR 4-9), respectively.

Overall, 4\% (8 patients) underwent open SRP while $92 \%$ (169) underwent robotic SRP and $4 \%$ (8) had laparoscopic SRP. Use of robotic surgery increased over time. All men undergoing SRP after FT had surgery performed robotically compared with $82 \%$ after RT. Of the men undergoing SRP after FT $74 \%$ had some form of nerve sparing (36\% unilateral, $32 \%$ bilateral, $6 \%$ incremental) compared to $10 \%$ after RT (7\% unilateral, 3\% bilateral).

Prior to SRP, there was no difference in patient age or pre-SRP PSA. However, patients undergoing SRP after RT had higher pre-primary, pre-SRP and final surgical pathology ISUP grading.

\section{Primary Outcome}

Toxicity. The toxicity of SRP is demonstrated in table 3. No significant differences in intraoperative complication rates were noted between men undergoing

Table 3. Summary and comparison of toxicity in focal therapy versus radiotherapy salvage prostatectomies

\begin{tabular}{lrrrrr}
\hline & \multicolumn{2}{c}{$\begin{array}{c}\text { SRP after } \\
\text { RT/BT }\end{array}$} & $\begin{array}{c}\text { SRP } \\
\text { after FT }\end{array}$ & p Value \\
\hline No. pts & 90 & & 95 & & 0.24 \\
No. intraop complications (\%): & 2 & $(2)$ & 0 & $(0)$ & \\
$\quad$ Ureteric injury & 1 & $(1)$ & 0 & $(0)$ & \\
Rectal injury & 1 & $(1)$ & 0 & $(0)$ & \\
No. postop complications (\%): & 31 & $(34)$ & 5 & $(5)$ & $<0.001$ \\
Anastomotic leak & 4 & $(4)$ & 1 & $(1)$ & \\
Anastomotic stricture & 11 & $(12)$ & 0 & $(0)$ & \\
Hematoma & 1 & $(1)$ & 1 & $(1)$ & \\
Metal clip migration & 1 & $(1)$ & 0 & $(0)$ & \\
Hernia requiring operation & 1 & $(1)$ & 0 & $(0)$ & \\
Postop ileus & 2 & $(2)$ & 2 & $(2)$ & \\
Intra-abdominal infection & 2 & $(2)$ & 0 & $(0)$ & \\
Prolonged catheter & 7 & $(8)$ & 0 & $(0)$ & \\
Wound infection & 1 & $(1)$ & 0 & $(0)$ & \\
Transient ischemic attack & 1 & $(1)$ & 0 & $(0)$ & \\
Diarrhea & 0 & $(0)$ & 1 & $(1)$ & \\
\hline
\end{tabular}

SRP after FT and those undergoing SRP after RT (0\% FT vs $2 \%$ RT). Postoperative complications within the first 30 days were significantly higher in men undergoing SRP after RT (table 3). These differences were preserved when analyzing patients who underwent SRP within 5 years of primary therapy (6\% FT vs $33 \% \mathrm{RT}$ ) and those undergoing SRP after 2015 (5\% FT vs 36\% RT). Postoperative complication rates within the RT group between EBRT and BT were similar (36\% EBRT vs $32 \% \mathrm{BT}, \mathrm{p}=0.82$ ).

Of the men undergoing SRP after RT 4\% experienced an anastomotic leak while $10 \%$ experienced an anastomotic stricture. Comparative figures for SRP after FT were $1 \%$ and $0 \%$, respectively. The RT group had more severe complications overall with 19\% experiencing a Clavien-Dindo grade IIIa/b complication compared to $1 \%$ of the FT group (fig. 1). In the RT group 1 patient experienced a rectal injury and 1 patient experienced a ureteric injury, both undergoing SRP after EBRT. Overall postoperative complication rates were not significantly different between minimally invasive and open surgical approaches (33\% vs $50 \%, \mathrm{p}=0.35$ ). However, anastomotic stricture rates were significantly lower with minimally invasive surgery (4\% [5 patients] vs $50 \%$ [4 patients], $\mathrm{p}<0.001$ ).

Functional. Men undergoing SRP after FT had significantly better continence at 12 months with $83 \%$ (64 patients) being pad-free while only $49 \%$ (38) of men undergoing SRP after RT were pad-free at 12 months $(\mathrm{p}<0.001)$. These differences were preserved when analyzing patients who underwent SRP within 5 years of primary therapy (79\% FT vs $50 \%$ RT; $\mathrm{p}=0.01)$ and those undergoing SRP after $2015(83 \%$ FT vs $42 \% \mathrm{RT}$; $\mathrm{p}<0.001)$.

Of men undergoing SRP after RT 18\% (14) were using 1 pad per day and 6\% were using 2 pads per day. There was no difference in continence rates between EBRT and BT ( $43 \%$ vs $57 \%, \mathrm{p}=0.25$ ).

Pre-salvage surgery potency rates were similar in both groups (67\% FT vs $65 \% \mathrm{RT} ; \mathrm{p}=0.94)$. Potency outcomes were similar between the 2 groups (14\% FT vs $11 \% \mathrm{RT}, \mathrm{p}=0.61$ ). The results were maintained when analyzing patients who underwent SRP within 5 years of primary therapy (12\% FT vs $10 \% \mathrm{RT} ; \mathrm{p}=1.0)$ and those undergoing SRP after 2015 (15\% FT vs 9\% RT; $p=0.52)$.

\section{Secondary Outcome: Oncological}

Median followup was 29.5 months (range 1.6-139.3). On univariate analysis overall 3-year BCR was similar between the 2 groups (35\% RT vs $32 \%$ FT, $\mathrm{p}=0.25$ ). Findings were similar when analyzing patients who underwent SRP within 5 years of primary therapy (33\% FT vs 47\% RT; p=0.26) and those undergoing SRP after 2015 (33\% FT vs $22 \%$ RT; $\mathrm{p}=0.25$ ). Only $13 \%$ of men had a positive margin in 
a RT SRP Post-operative Complications

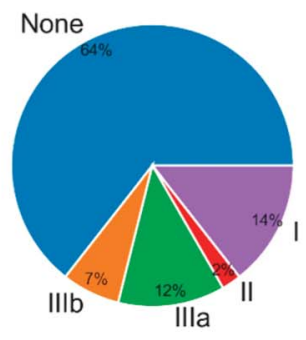

Clavien-Dindo Grade b

\section{FT SRP Post-operative Complications}



Clavien-Dindo Grade

Figure 1. Comparison of postoperative complication rates in 185 salvage radical prostatectomies after radiation therapy and focal therapy by Clavien-Dindo grade.

the FT group (2 with T2, 5 with T3a, 4 with T3b) versus $37 \%$ in the RT group (6 with T2, 9 with T3a, 15 with T3b). There was no difference in 3-year BCR between SRP after EBRT vs BT (31\% vs $35 \%$, $\mathrm{p}=0.64$ ). Kaplan-Meier curves were not significantly different (figure 2, log-rank test: $\mathrm{p}=0.67$ ).

Two multivariate Cox regression models were constructed, one adjusting for risk factors prior to primary therapy (supplementary table 2, https:// www.jurology.com) and another adjusting for risk factors prior to SRP (table 4). On multivariate analysis SRP after RT was associated with lower BCR compared with SRP after FT (HR 0.36, 95\% CI $0.18-0.82, \mathrm{p}<0.005)$.

\section{DISCUSSION}

The current multicenter cohort study demonstrates that men undergoing SRP after FT experience lower postoperative complication rates and better urinary continence outcomes. Oncological outcomes in terms of positive margin rates were better in men undergoing SRP after FT whereas BCR was lower in multivariable analysis for men undergoing SRP after RT.

\section{Comparison with Other Studies}

To our knowledge only 1 other study has compared the toxicity and oncological outcomes of SRP after radiation with SRP after FT. Onol and colleagues have recently reported on 94 men undergoing SRP after radiation and a further 32 men undergoing SRP after focal ablation therapy. ${ }^{12}$ The study design is similar to the current study but with fewer patients and represents the outcomes of a single institution and high-volume surgeon. That said, their conclusions match those of the current study. They report functional outcomes of SRP after radiation inferior to those of salvage surgery after FT and similar

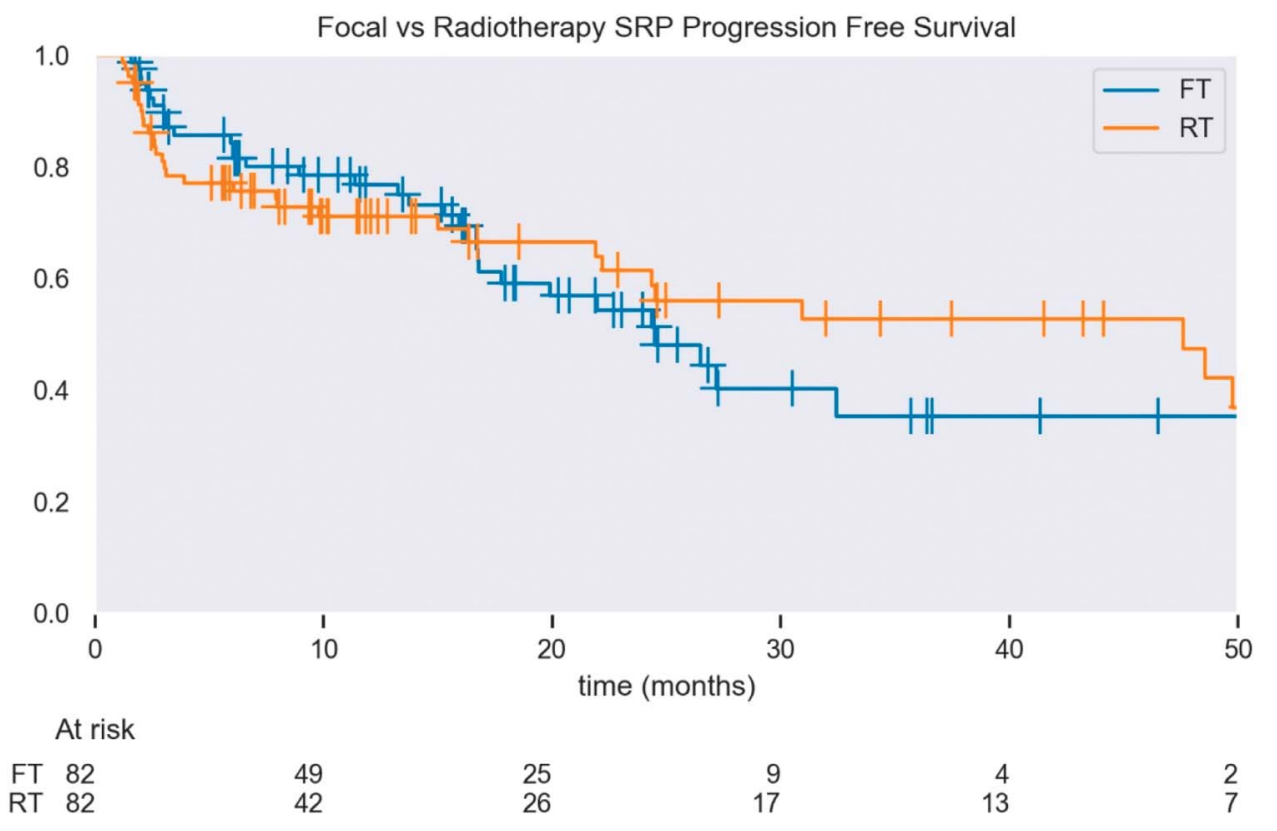

Figure 2. Kaplan-Meier curves of progression-free survival by biochemical recurrence after focal therapy and radiotherapy salvage prostatectomies in 164 patients. Log-rank test: $p=0.67$. 
Table 4. Multivariate Cox proportional hazards regression model to evaluate perioperative risk factors for biochemical recurrence in salvage radical prostatectomies

\begin{tabular}{lccc}
\hline & \multicolumn{2}{c}{ BCR (Outcome) } & \\
\cline { 2 - 3 } & HR & $95 \%$ Cl & p Value \\
\hline Age & 0.98 & $0.94-1.02$ & 0.33 \\
Pre-prostatectomy PSA & 1.01 & $0.97-1.05$ & 0.66 \\
Primary therapy: & & & \\
$\quad$ Focal therapy & Reference & Reference & Reference \\
$\quad$ Radiotherapy & 0.51 & $0.26-0.98$ & 0.04 \\
Pathological stage: & & & \\
pT2 & Reference & Reference & Reference \\
pT3a & 1.56 & $0.77-3.15$ & 0.21 \\
pT3b & 2.86 & $1.35-6.03$ & 0.01 \\
Preop ISUP grade: & & & \\
ISUP 1 + 2 & Reference & Reference & Reference \\
ISUP 3, 4, 5 & 1.10 & $0.62-1.95$ & 0.75 \\
Postop ISUP grade: & & & \\
$\quad$ ISUP 1 + 2 & Reference & Reference & Reference \\
ISUP 3, 4, 5 & 1.26 & $0.64-2.47$ & 0.51 \\
Positive margin & 1.39 & $0.75-2.59$ & 0.29 \\
Procedure yr & 0.95 & $0.84-1.08$ & 0.42 \\
\hline
\end{tabular}

oncological outcomes. They also report occurrence of nonorgan confined disease and positive margin rates higher in men with surgery after FT compared to after radiation, a finding at odds with our current study.

Toxicity. In the current study, intraoperative toxicity of salvage surgery was low and did not differ between men with previous radiation or previous focal therapy. We report 2 intraoperative injuries, namely 1 ureteric and 1 rectal injury. Yuh et al have previously reported a similar incidence of ureteric injury in their series of 51 men undergoing salvage robotic radical prostatectomy after $\mathrm{RT} \mathrm{.}^{13}$ A recent systematic review on SRP after RT by Chade et al reported rectal injury rates between $0 \%$ and $28 \%{ }^{14}$ The review reported a trend towards lower rate of intraoperative complications which may be related to increased use of robotic surgery. This could explain our low reported rectal injury and overall complication rate as the majority of our cases were performed robotically. ${ }^{13,15,16}$

The incidence of anastomotic complications seen in the current study was similar to a recent systematic review of complications following SRP that reported between $7 \%$ and $41 \%$ experienced anastomotic strictures after surgery while a further $14 \%$ to $18 \%$ experienced an anastomotic leak. ${ }^{14}$

Functional. We found that men undergoing surgery after FT required fewer continence pads at 12 months than men with previous RT. Onol and colleagues report similar findings with $77 \%$ of men pad-free 12 months after SRP for FT recurrence versus 39\% for men who underwent SRP for radiation failure. ${ }^{12}$ Comparative figures in the current study were $83 \%$ and $49 \%$, respectively. A recent systematic review found between $20 \%$ and $90 \%$ of men reported urinary incontinence, however on average around $50 \%$ reported some degree of urinary incontinence at 12 months, similar to the current study. ${ }^{17}$

It is unclear why men who underwent salvage surgery after RT had worse continence outcomes than after FT. However, causes of urinary incontinence after radical prostatectomy are multifactorial, ${ }^{18}$ including detrusor overactivity, poor bladder compliance and intrinsic sphincter deficiency. It may be that prior radiation therapy increases the incidence of these such that the patients with prior FT have lower rates of bladder and sphincter dysfunction prior to salvage surgery.

Men undergoing SRP after RT were less likely to undergo nerve sparing surgery, however potency outcomes at 12 months were similar in both groups. Potency is known to continue to improve 2 to 3 years after prostate cancer surgery, and therefore longer followup may reveal the impact of nerve sparing surgery. ${ }^{19}$

Oncological. We report a 3-year BCR of 34\% and 32\% for men undergoing SRP after FT and SRP after RT, respectively, a little lower than that reported in a systematic review. ${ }^{14}$ Despite the high rate of locally advanced disease, it was reassuring that our positive margin rate is lower than in many salvage prostatectomy series. ${ }^{14,20,21}$ Why we found significantly worse BCR for men undergoing SRP after FT within our multivariable model raises several questions, especially as these men had a lower rate of positive margins and lower final pathological stage. It may be that men with prior FT are more likely to have micrometastatic disease that was not identified on pre-surgery imaging compared to men undergoing SRP after RT. However, this hypothesis is at odds with the lower stage disease found on final pathological analysis.

\section{Clinical Implications}

Traditionally SRP has been associated with significant functional toxicity. However, we demonstrate that the functional outcome of SRP is not universally poor and is dependent on primary prostate cancer treatment. Therefore, we would encourage urologists reviewing men with recurrent prostate cancer after FT to consider salvage surgery as an alternative to salvage radiotherapy or whole gland ablation. Furthermore, by performing SRP on men with recurrent prostate cancer after FT, these men still have radiation to the prostate bed in reserve if the SRP is not curative.

The relatively high $\mathrm{BCR}$ rate for men undergoing SRP after FT suggests that all men considering SRP after FT should undergo cross-sectional imaging, utilizing, for example, prostate specific membrane antigen positron emission tomography imaging, to 
exclude micrometastatic prostate cancer. Furthermore, men should be fully counseled that SRP after FT may be part of a multimodal approach to the treatment of their disease.

\section{Strengths and Limitations}

The present study is limited by the retrospective nature of data collection, similar to many previous studies reporting on salvage prostatectomy. Due to the retrospective design, we were unable to report standardized patient reported outcomes such as Expanded Prostate Cancer Index Composite (EPIC)/ International Index of Erectile Function (IIEF-5). However, we have used very strict definitions of functional return: continent if they report 0 pads required at 12 months and potent if they report ability to perform penetrative intercourse.

Another limitation concerns the variation in surgical approach between the 2 groups. A recent randomized study of robotic versus open primary radical prostatectomy demonstrated no difference in functional and oncological outcome between the 2 surgical approaches, though this may not apply in the salvage setting. ${ }^{22}$

Significant differences in patient characteristics between men undergoing SRP after FT and men undergoing SRP after RT in terms of stage and grade of disease could have impacted our findings, including high $\mathrm{pNx}$ rates. Proportionally more men underwent SRP after RT at the beginning of the study period compared to after FT, which could imply a learning curve. While propensity score matching was not possible due to sample size, multivariable analysis and subgroup analysis for all outcomes have largely adjusted for these differences.

\section{CONCLUSIONS}

This multicenter retrospective cohort study demonstrates that SRP perioperative complications and long-term urinary continence recovery are dependent on primary prostate cancer therapy, with men undergoing SRP after FT experiencing lower postoperative complication rates and better urinary continence outcomes.

\section{REFERENCES}

1. Valerio $\mathrm{M}$, Ahmed $\mathrm{HU}$, Emberton $\mathrm{M}$ et al: The role of focal therapy in the management of localised prostate cancer: a systematic review. Eur Urol 2014; 66: 732.

2. Haj-Hamed M, Karivedu V and Sidana A: Salvage treatment for radio-recurrent prostate cancer: a review of literature with focus on recent advancements in image-guided focal salvage therapies. Int Urol Nephrol 2019; 51: 1101

3. Guillaumier S, Peters $M$, Arya $M$ et al: A multicentre study of 5-year outcomes following focal therapy in treating clinically significant nonmetastatic prostate cancer. Eur Urol 2018; 74: 422.

4. Tourinho-Barbosa RR, Sanchez-Salas R, Claros $\mathrm{OR}$ et al: Focal therapy for localized prostate cancer with either high intensity focused ultrasound or cryoablation: a single institution experience. J Urol 2020; 203: 320.

5. Kaffenberger SD and Smith JA: Salvage robotic radical prostatectomy. Indian J Urol 2014; 30: 429.

6. Agarwal PK, Sadetsky N, Konety BR et al: Treatment failure after primary and salvage therapy for prostate cancer. Cancer 2008; 112: 307.

7. Rogers $E$, Ohori $M$, Kassabian VS et al: Salvage radical prostatectomy: outcome measured by serum prostate specific antigen levels. J Urol 1995; 153: 104.

8. Chade DC, Shariat SF, Cronin AM et al: Salvage radical prostatectomy for radiation-recurrent prostate cancer: a multi-institutional collaboration. Eur Urol 2011; 60: 205.
9. Paparel P, Cronin AM, Savage C et al: Oncologic outcome and patterns of recurrence after salvage radical prostatectomy. Eur Urol 2009; 55: 404.

10. Stabile A, Orczyk C, Hosking-Jervis $F$ et al: Medium-term oncological outcomes in a large cohort of men treated with either focal or hemiablation using high-intensity focused ultrasonography for primary localized prostate cancer. BJU Int 2019; 124: 431

11. Marconi L, Stonier T, Tourinho-Barbosa R et al: Robot-assisted radical prostatectomy after focal therapy: oncological, functional outcomes and predictors of recurrence. Eur Urol 2019; 76: 27.

12. Onol FF, Bhat S, Moschovas M et al: Comparison of outcomes of salvage robot-assisted laparoscopic prostatectomy for post-primary radiation vs focal therapy. BJU Int 2020; 125: 103.

13. Yuh B, Ruel N, Muldrew S et al: Complications and outcomes of salvage robot-assisted radical prostatectomy: a single-institution experience. BJU Int 2014; 113: 769 .

14. Chade DC, Eastham J, Graefen M et al: Cancer control and functional outcomes of salvage radical prostatectomy for radiation-recurrent prostate cancer: a systematic review of the literature. Eur Urol 2012; 61: 961.

15. Kaffenberger SD, Keegan KA, Bansal NK et al: Salvage robotic assisted laparoscopic radical prostatectomy: a single institution, 5-year experience. J Urol 2013; 189: 507.
16. Zugor $V$, Labanaris $A P$, Porres $D$ et al: Robotassisted radical prostatectomy for the treatment of radiation-resistant prostate cancer: surgical, oncological and short-term functional outcomes. Urol Int 2014; 92: 20.

17. Zargar H, Lamb AD, Rocco B et al: Salvage robotic prostatectomy for radio recurrent prostate cancer: technical challenges and outcome analysis. Minerva Urol Nefrol 2017; 69: 26.

18. Hoyland K, Vasdev N, Abrof A et al: Post-radical prostatectomy incontinence: etiology and prevention. Rev Urol 2014; 16: 181.

19. Prabhu V, Lee T, McClintock TR et al: Short-, intermediate-, and long-term quality of life outcomes following radical prostatectomy for clinically localized prostate cancer. Rev Urol 2013; 15: 161.

20. Ogaya-Pinies G, Linares-Espinos E, HernandezCardona $E$ et al: Salvage robotic-assisted radical prostatectomy: oncologic and functional outcomes from two high-volume institutions. World J Urol 2019; 37: 1499.

21. Linares Espinós E, Sánchez-Salas R, Sivaraman A et al: Minimally invasive salvage prostatectomy after primary radiation or ablation treatment. Urology 2016; 94: 111.

22. Coughlin GD, Yaxley JW, Chambers SK et al: Robot-assisted laparoscopic prostatectomy versus open radical retropubic prostatectomy: 24-month outcomes from a randomised controlled study. Lancet Oncol 2018; 19: 1051. 


\section{EDITORIAL COMMENTS}

The advent of magnetic resonance imaging (MRI) guided biopsy has led to increased finding of intermediate risk prostate cancers; more than half of all cancers diagnosed with the new method are of ISUP Groups 2 and 3 (see figure). Because men with intermediate risk cancers are most suitable for partial gland ablation (PGA), the candidate pool for such treatment is growing. When considering options, one question PGA candidates frequently ask is, "if focal therapy fails, can I still get safe and effective

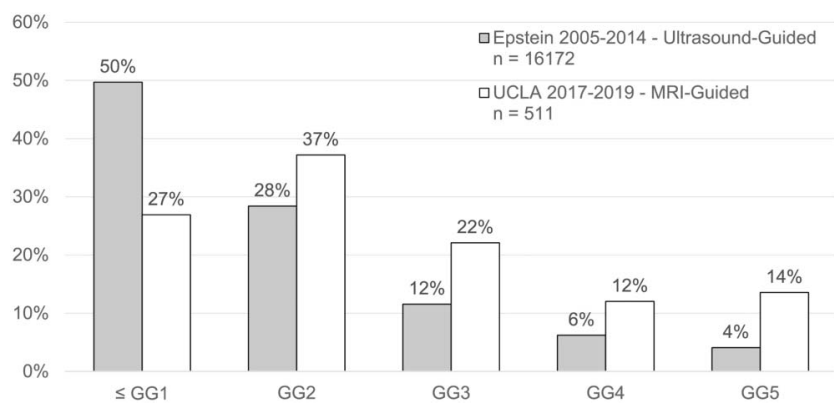

Prostate biopsy findings (Gleason grade group [GG]) before and after advent of MRI guidance. In earlier series men were biopsied with ultrasound guidance (closed bars); in contemporary series men were diagnosed with MRI guided biopsy (open bars). Note that $59 \%$ of all cancers currently diagnosed are of intermediate risk (ISUP 2 or 3 ) and may be considered possible candidates for focal therapy. From Chuang $R$ et al: Hemigland cryoablation of clinically significant prostate cancer: intermediate-term followup via magnetic resonance imaging guided biopsy. J Urol 2020; 204: 941. surgery later on?" The present work by Ribeiro and associates helps answer the question in the affirmative.

In Ribeiro et al's large retrospective analysis, salvage prostatectomy after PGA (95 patients) was much better tolerated than after radiation therapy (90). Therefore, the favorable results after focal therapy (only 5\% complication rate in first 30 days and $83 \%$ continence rate at 1 year) will provide some reassurance that a reasonable fallback option exists. The poor results following radiation-salvage surgery have become well known; thus, surgery after failed radiation is no longer a routine option. For that reason, a more relevant comparison, de novo surgery vs post-PGA surgery, would provide the kind of information that focal therapy candidates seek. The limited studies currently available suggest results may be comparable. ${ }^{1}$

Despite lack of prospective, clinical trial data, adoption of PGA is increasing rapidly, ${ }^{1}$ at least partly because patients with prostate cancer generally prioritize quality of life in making treatment decisions. ${ }^{2}$ However, PGA may fail to ablate clinically significant cancer in $20 \%$ of cases. Thus, evaluation of PGA-salvage treatments will become an important research mandate in the near future.

Leonard S. Marks* and Wayne G. Brisbane

Department of Urology

University of California-Los Angeles

Los Angeles, California

*Financial and/or other relationship with Avenda Health.

\section{REFERENCES}

1. Marra G, Valerio M, Emberton M et al: Salvage local treatments after focal therapy for prostate cancer. Eur Urol Oncol 2019; 2: 526.

2. Watson V, McCartan N, Krucien N et al: Evaluating the trade-offs men with localized prostate cancer make between the risks and benefits of treatments: the COMPARE study. J Urol 2020; 204: 273.

This multicenter, nonrandomized, retrospective study of 185 patients compares 30-day complications along with 12-month functional and oncological outcomes of SRP after RT vs FT. The authors demonstrate fewer and less severe 30-day complications for SRP after primary FT compared to RT, particularly in regard to anastomotic leak or stricture. SRP after FT has a greater rate of urinary continence at 1 year with $83 \%$ requiring no pads. This may not be surprising as FT is more targeted while RT often induces obliterative endarteritis with scatter effect on the urinary sphincter and surrounding tissues. Men undergoing SRP after RT were less likely to have nerve-sparing surgery yet the potency rates were similarly low for both groups (14\% FT, $11 \%$ RT), although the followup was short at 12 months. It is not clear why SRP after FT had 
significantly higher BCR despite lower stage/ grade and positive margins compared to the RT group.

One limitation is that although these were consecutive patients, the patients undergoing RT were from an earlier era and not contemporaneous, arguing that perhaps newer RT methods might improve the outcomes. Regarding SRP, the rate of anastomotic stricture was significantly lower in men who underwent a minimally invasive approach. Overall, this is good news for SRP after FT with less salvage toxicity, but the reader should keep in mind that there are also other salvage options that may have a potentially lower toxicity profile, namely whole gland ablation or salvage RT after FT failure.

Thomas J. Polascik

Duke Cancer Institute Durham, North Carolina 